\begin{tabular}{|c|c|c|}
\hline Case Reports in & \multicolumn{2}{|c|}{ Case Rep Gastroenterol 2014;8:186-192 } \\
\hline Gastroenterology & $\begin{array}{l}\text { DOI: 10.1159/000363177 } \\
\text { Publisned onIIne: IVlay } 15,2014\end{array}$ & $\begin{array}{l}\text { (c) } 2014 \text { S. Karger AG, Basel } \\
1662-0631 / 14 / 0082-0186 \$ 39.50 / 0 \\
\text { www.karger.com/crg }\end{array}$ \\
\hline & $\begin{array}{l}\text { This is an Open Access article licer } \\
\text { NonCommercial } 3.0 \text { Unported lice } \\
\text { the online version of the article onl }\end{array}$ & $\begin{array}{l}\text { s of the Creative Commons Attribution } \\
\text { w.karger.com/OA-license), applicable to to } \\
\text { tted for non-commercial purposes only. }\end{array}$ \\
\hline
\end{tabular}

\title{
Perianal Paget's Disease Co-Associated with Anorectal Adenocarcinoma: Primary or Secondary Disease?
}

\author{
Xiujun Liao ${ }^{\mathrm{a}} \quad$ Weiming $\mathrm{Mao}^{\mathrm{a}} \quad \mathrm{A}^{\prime} \mathrm{Li}_{\text {Lin }}^{\mathrm{b}}$ \\ Departments of ${ }^{a}$ Colorectal Surgery and ${ }^{b}$ Pathology, The 3rd People's Hospital of \\ Hangzhou, Hangzhou, China
}

\section{Key Words}

Perianal Paget's disease · Anorectal adenocarcinoma - Cytokeratin 7 - Gross cystic disease fluid protein $15 \cdot$ Cytokeratin 20

\begin{abstract}
Perianal Paget's disease (PPD) represents a skin neoplasm which can be either primary or secondary to carcinoma from an adjacent internal organ. PPD with underlying colorectal adenocarcinoma is usually looked upon as a secondary disease. We report a rare case of coassociated PPD and anorectal adenocarcinoma. The PPD was found to be located near the anorectal adenocarcinoma with normal tissues between them. Immunohistochemical stains demonstrated that the Paget's cells were CK7+/GCDFP-15-/CK20-/MUC2-/CDX2-, whereas the anorectal adenocarcinoma was shown to be CK7+/GCDFP-15-/CK20+/MUC2+/CDX2+. This immunological phenotypic profile supported the notion that PPD and anorectal adenocarcinoma were of different origins, but could not define the exact origins of PPD. In our determination, this case was a primary PPD with anorectal adenocarcinoma. PPD remains a heterogeneous and complex pathology, and additional studies are required to differentiate between the various possible origins.

(c) 2014 S. Karger AG, Basel
\end{abstract}

\section{Introduction}

Perianal Paget's disease (PPD) is a variant of extramammary Paget's disease (EMPD) and represents a rare intraepidermal adenocarcinoma characterized by the presence of typical Paget's cells, appearing as large rounded vacuolated cells. Underlying associated

Xiujun Liao

Department of Colorectal Surgery

The 3rd People's Hospital of Hangzhou

Hangzhou 310009 (China)

E-Mail liaoxiujun@126.com 
Liao et al.: Perianal Paget's Disease Co-Associated with Anorectal Adenocarcinoma: Primary or Secondary Disease?

colorectal adenocarcinoma is a frequent comorbidity, and approximately $40-60 \%$ of patients with PPD harbor a colorectal neoplasm [1, 2]. PPD with underlying adenocarcinoma usually represents an intraepidermal extension of an invasive carcinoma from an adjacent internal organ, so this kind of PPD is generally looked upon as a secondary disease. From the detection of immunohistochemical markers, such as cytokeratin 7 (CK7), CK20, and gross cystic disease fluid protein 15 (GCDFP-15), PPD can be distinguished as a primary or a secondary disease. Here, we report a rare case of synchronous PPD and anorectal adenocarcinoma. Initially, we thought that PPD was a secondary disease due to its proximity to the carcinoma. However, the immunohistochemical staining patterns did not support it. This case suggests that we identified a primary PPD, which was co-associated with anorectal adenocarcinoma.

\section{Case Report}

A 78-year-old male was referred to our colorectal department with a more than 1-year history of perianal irritation and pain. He complained that these symptoms troubled him almost every day and worsened during defecation. He thought they decreased his quality of his life significantly, though he could endure the mild symptoms. There was no evidence of perirectal bleeding. He was diagnosed as suffering from hemorrhoids by the community physicians and was treated with a topical cream named compound carraghenates cream, $0.5 \mathrm{~g}$, three times a day. However, there were no improvement. He had no weight loss or any other gastrointestinal symptoms. He had no family history of colon cancer or other malignancy. He had had type II diabetes for 15 years, glucose levels being controlled by metformin tablets. He had had hypertension (stage 2) for 3 years. His blood pressure was in the normal range by take one tablet of Norvasc per day. Physical examination showed pale plaque-like lesions involving two sides and the posterior zone of the perianal skin, measuring $1 \times 3 \mathrm{~cm}$, with no evidence of erythema or anabrosis. Digital rectal examination of the anus and rectum revealed a $1.0 \times 1.2 \mathrm{~cm}$ tumor located in the posterior anorectal region in close proximity to the perianal lesion. The tumor was hard with tenderness and good mobility. There was no evidence of inguinal lymphadenopathy.

In the preoperative setting, a colonoscopy was done and showed slight inflammation of the rectum. A CT scan of his chest, abdomen and pelvis was unremarkable. Endoanal ultrasound showed evidence of a tumor within the anal canal without invasion of the surrounding anal sphincter musculature. Biopsy of the tumor and perianal skin under local anesthesia was performed. Histological examination of the specimen was suggestive of a malignant tumor of epidermal origin, possibly representing carcinoma of the sweat gland.

As the patient had refused abdominoperineal resection and the tumor might be syringocarcinoma, wide local excision and reconstruction with transposition flap was performed. However, histological examination of the resected specimen revealed a poorly differentiated anorectal adenocarcinoma and PPD (fig. 1). The margins of both lesions were clear of malignancy. Immunohistochemical stains were positive for CK7, but negative for GCDFP-15, CK20, CDX2 and MUC2 in the Paget's cells. CK7, CK20, CDX2 and MUC2, but not GCDFP-15, were present in cells of the invasive anorectal adenocarcinoma (fig. 2). We strongly advised the patient to receive abdominoperineal resection or chemoradiotherapy. However, he declined further treatment and was discharged 2 weeks after the operation. A 6-month follow-up showed no recurrence of Paget's disease or of the adenocarcinoma. 
Liao et al.: Perianal Paget's Disease Co-Associated with Anorectal Adenocarcinoma: Primary or Secondary Disease?

\section{Discussion}

Paget's disease is a rare cutaneous disorder described as an apocrine gland tumor occurring in both a benign and a malignant form, with metastatic potential, which was first reported in the breast by Sir James Paget in 1874. Since then, EMPD has been reported in several extramammary sites, including the axilla, thigh, groin, perineum, scrotum and vulva [3]. When EMPD affects the perianal region, it is called PPD. The first case of PPD was reported by Darrier and Couillaud in 1893 [4].

PPD is an uncommon condition of the perianal region, and its incidence is higher in persons between 50 and 70 years of age $[5,6]$. Microscopically, typical histological features of large, round and clear-staining cells with large nuclei characterize PPD. In some cases of PPD, signet ring cells with a pale, vacuolated cytoplasm are also present. In some lesions both cells can be found, while in others one type of cell predominates.

PPD with an underlying malignancy of digestive origin is rare. Until the end of 2012, at least to the best of our knowledge, there were fewer than 60 cases reported in the English language literature [7]. Primary PPD occurs more commonly in females [6], while PPD with underlying colorectal carcinoma is more common in males (male:female ratio $=17: 8$ ) [7].

There are four main hypotheses proposed to explain the origins of PPD [7, 8]. First, Paget's cells may arise from an underlying carcinoma of eccrine or apocrine glands, especially syringocarcinoma, with a secondary epidermal involvement. Second, Paget's cells might be metastatic from underlying carcinoma cells. Third, it could be due to simultaneous neoplastic changes in the epidermis, apocrine structures and glandular elements of the rectum. There is one last hypothesis, which would explain those cases without malignancies. Paget's cells may arise from pluripotent ectodermal basal cells resulting in adenocarcinoma in situ, with a long pre-invasive phase [3, 9].

Despite PPD being a heterogeneous disease, there are generally two main types of PPD. One is primary PPD, which originates from the epidermis or skin appendages, including eccrine gland, apocrine gland, ectopic mammary-like gland, epidermal pluripotent stem cells and other structures. The other is secondary PPD, also known as 'pagetoid phenomena', which is a metastatic tumor that is derived from underlying carcinoma cells.

Immunohistochemistry is very useful in differentiating the type of PPD. Frequently used immunohistochemical markers were CK7, GCDFP-15 and CK20. CK7 is a sensitive marker for almost all pagetoid neoplasms of the breast and genital skin, but is also expressed by some rectal adenocarcinomas. Thus, CK7 has no practical value to distinguish the type of PPD [10, 11]. Both GCDFP-15 and CK20 are helpful in identifying secondary PPD. GCDFP-15 is considered as apocrine epithelium-specific tissue marker. Wick et al. [12] reported that GCDFP-15 was a marker for breast cancer. However, there were no incidences in expression of GCDFP-15 in 22 cases of rectal cancer. Hence GCDFP-15 is usually positive in breast cancer and primary PPD cases [13-15]. Expression of CK20 might be seen in colorectal carcinomas but not in primary PPD [16]. Rectal carcinomas often display the immunophenotype CK7-/CK20+/GCDFP-15-, while for PPD CK7-/CK20+/GCDFP-15- pattern of expression is the most frequently associated phenotype. If PPD displays CK7+/CK20-/ GCDFP-15+ expression, this pattern of phenotypic expression is considered primary disease.

Recently, the evaluation of the gene expression of tissue mucin (MUC2) and CDX2 has been advocated to be a useful tool in differentiating primary and secondary PPD. MUC2 is always found in gastrointestinal mucosa and secondary perianal PPD [17]. CDX2 is considered specific for enterocytes and has been used for the diagnosis of primary and metastatic colonic adenocarcinoma. It appears to be specific for cutaneous metastases from intestinal and urothelial carcinomas and is a useful diagnostic marker of these tumors, being 
Liao et al.: Perianal Paget's Disease Co-Associated with Anorectal Adenocarcinoma: Primary or Secondary Disease?

especially useful in the diagnosis of EMPD associated with an underlying colorectal tumor [18].

We used five immunohistochemical markers to identify the type of PPD. The immunohistochemical characteristics were determined to be CK7+/GCDFP-15-/CK20-/MUC2-/ CDX2-, while the anorectal adenocarcinoma was determined to be CK7+/GCDFP-15-/ CK20+/MUC2+/CDX2+. From the results, we can conclude that PPD and anorectal adenocarcinoma are separate entities. Expression of CK20, CDX2 and MUC2 are markers suggestive of rectal carcinoma, yet they were all absent in the Paget's cell of our case study. This was a rare phenomenon. At least from the available literature, markers of rectal carcinoma, especially CK20, were always positive in cases of PPD with underlying anorectal carcinoma, which therefore gave the appearance of a secondary disease to the concurrent carcinoma. In 2009, Chanjuan and Argani [19] reported a case with synchronous PPD and rectal adenocarcinoma, which showed an immunohistochemical pattern of expression as CK7+/CK20-/ GCDFP-15+ in Paget's cells and CK7+(variable)/CK20+/GCDFP-15- in rectal adenocarcinoma cells. The authors believed that this was the first reported case of primary PPD with primary rectal carcinoma. We suggest that our case is the second one to be reported. With the exception of CK20, our case expressed another two markers (CDX2 and MUC2) to highly suggest that PPD was not homologous with anorectal cancer. However, GCDFP-15 was negative in our case. GCDFP-15 is the marker for the apocrine gland [20], so it is difficult to define the exact origin of PPD of our case. If it was not secondary to the anorectal adenocarcinoma and had not developed from the apocrine gland, which department of the epidermis was its exact origin? Until now, we do not have sufficient information to answer this question.

From the clinical perspectives of PPD and anorectal adenocarcinoma, we would like to consider PPD as a secondary disease. We could explain the immunohistochemical observations by hypothesizing that the cells of rectal adenocarcinoma migrated throughout the skin and altered their morphology. Mai [21] reported the theory of 'field effect' as a mechanism for tumor metastasis in mammary Paget's disease. The microenvironment of perianal epithelium might induce the loss of typical cellular characteristics, which spread from anorectal carcinoma. In fact the transition of Paget's cell did exist. Grelck et al. [22] reported a case in whom they captured the transition from PPD to invasive carcinoma. In addition, Matin et al. [23] reported a case of primary cutaneous mucinous carcinoma arising on a background of EMPD of the vulva and perineum. With regard to our case, there is still a lack of evidence supporting the notion that PPD was derived from anorectal adenocarcinoma and lost the expression of CK20, CDX2 and MUC2 in the environment of the perianal epithelium.

\section{Conclusion}

We report a primary case of PPD with anorectal adenocarcinoma based on their discordant immunostaining expression patterns. The risk of recurrence and metastasis was high because the patient just received local excision instead of radical operation. The PPD likely had a different origin from that of the anorectal adenocarcinoma. However, the current immunophenotypic markers could not identify its possible origin. Therefore, PPD remains a heterogeneous and complex entity. Additional studies are clearly needed to help evaluate its origin, since further understanding its origin will certainly contribute to improved management of this complex entity. Radiotherapy and chemotherapy will benefit long-term survival and close follow-up is necessary. 


\begin{tabular}{l|l}
\hline Case Rep Gastroenterol 2014;8:186-192 \\
\hline DOI: 10.1159/000363177 & $\begin{array}{l}\text { C 2014 S. Karger AG, Basel } \\
\text { www.karger.com/crg }\end{array}$ \\
\hline
\end{tabular}

Liao et al.: Perianal Paget's Disease Co-Associated with Anorectal Adenocarcinoma: Primary or Secondary Disease?

\section{Disclosure Statement}

The authors declare that they have no competing interests.

\section{References}

1 Sahai A, Kodner IJ: Premalignant neoplasms and squamous cell carcinoma of the anal margin. Clin Colon Rectal Surg 2006;19:88-93.

-2 Leonard D, Beddy D, Dozois EJ: Neoplasms of anal canal and perianal skin. Clin Colon Rectal Surg 2011;24:54-63.

-3 Jones RE Jr, Austin C, Ackerman AB: Extramammary Paget's disease. A critical reexamination. Am J Dermatopathol 1979;1:101-132.

4 Darrier J, Couillaud P: Sur un cas de maladie de Paget de la région périnéo-anale et scrotale. Ann Dermatol Syphil 1893;4:25-31.

5 Beahrs OH, Wilson SM: Carcinoma of the anus. Ann Surg 1976;184:422-428.

6 Shepherd NA: Anal intraepithelial neoplasia and other neoplastic precursor lesions of the anal canal and perianal region. Gastroenterol Clin North Am 2007;36:969-987, ix.

-7 Pellino G, Sciaudone G, Canonico S, Selvaggi F: Perianal Paget's disease and malignancies of lower hindgut and anal canal. J Gastroenterol Hepatol Res 2012;1:1-4.

8 Marvin L. Corman: Colon \& Rectal Surgery. Philadelphia, Lippincott-Raven, 1998.

$\checkmark$ Beck DE, Fazio VW: Perianal Paget's disease. Dis Colon Rectum 1987;30:263-266.

10 Smith KJ, Tuur S, Corvette D, Lupton GP, Skelton HG: Cytokeratin 7 staining in mammary and extramammary Paget's disease. Mod Pathol 1997;10:1069-1074.

11 Lundquist K, Kohler S, Rouse RV: Intraepidermal cytokeratin 7 expression is not restricted to Paget cells but is also seen in Toker cells and Merkel cells. Am J Surg Pathol 1999;23:212-219.

12 Wick MR, Lillemoe TJ, Copland GT, Swanson PE, Manivel JC, Kiang DT: Gross cystic disease fluid protein-15 as a marker for breast cancer: immunohistochemical analysis of 690 human neoplasms and comparison with alpha-lactalbumin. Hum Pathol 1989;20:281-287.

13 Kohler S, Smoller BR: Gross cystic disease fluid protein-15 reactivity in extramammary Paget's disease with and without associated internal malignancy. Am J Dermatopathol 1996;18:118-123.

14 Goldblum JR, Hart WR: Perianal Paget's disease: a histologic and immunohistochemical study of 11 cases with and without associated rectal adenocarcinoma. Am J Surg Pathol 1998;22:170-179.

15 Ansai S, Mitsuhashi Y, Kondo S, Manabe M: Immunohistochemical differentiation of extra-ocular sebaceous carcinoma from other skin cancers. J Dermatol 2004;31:998-1008.

16 Chu P, Wu E, Weiss LM: Cytokeratin 7 and cytokeratin 20 expression in epithelial neoplasms: a survey of 435 cases. Mod Pathol 2000;13:962-972.

-17 Selvaggi F, Guadagni I, Pellino G, De Rosa M, Imbrogno G, Sciaudone G: Perianal Paget's disease happening with mucinous adenocarcinoma of the anal canal: managing rarities. J Cutan Pathol 2010;37:1182-1183.

18 Lora V, Kanitakis J: CDX2 expression in cutaneous metastatic carcinomas and extramammary Paget's disease. Anticancer Res 2009;29:5033-5037.

19 Chanjuan S, Argani P: Synchronous primary perianal Paget's disease and rectal adenocarcinoma: report of a hitherto undescribed phenomenon. Int J Surg Pathol 2009;17:42-45.

20 Viacava P, Naccarato AG, Bevilacqua G: Spectrum of GCDFP-15 expression in human fetal and adult normal tissues. Virchows Arch 1998;432:255-260.

21 Mai KT: Morphological evidence for field effect as a mechanism for tumour spread in mammary Paget's disease. Histopathology 1999;35:567-576.

-22 Grelck KW, Nowak MA, Doval M: Signet ring cell perianal Paget disease: loss of MUC2 expression and loss of signet ring cell morphology associated with invasive disease. Am J Dermatopathol 2011;33:616-620.

23 Matin RN, Gibbon K, Rizvi H, Harwood CA, Cerio R: Cutaneous mucinous carcinoma arising in extramammary Paget disease of the perineum. Am J Dermatopathol 2011;33:705-709. 


\begin{tabular}{|c|c|c|}
\hline \multirow{3}{*}{$\begin{array}{l}\text { Case Reports in } \\
\text { Gastroenterology }\end{array}$} & \multirow{2}{*}{\multicolumn{2}{|c|}{ Case Rep Gastroenterol 2014;8:186-192 }} \\
\hline & & \\
\hline & DOI: 10.1159/000363177 & $\begin{array}{l}\text { (c) } 2014 \text { S. Karger AG, Basel } \\
\text { www.karger.com/crg }\end{array}$ \\
\hline
\end{tabular}
Primary or Secondary Disease?
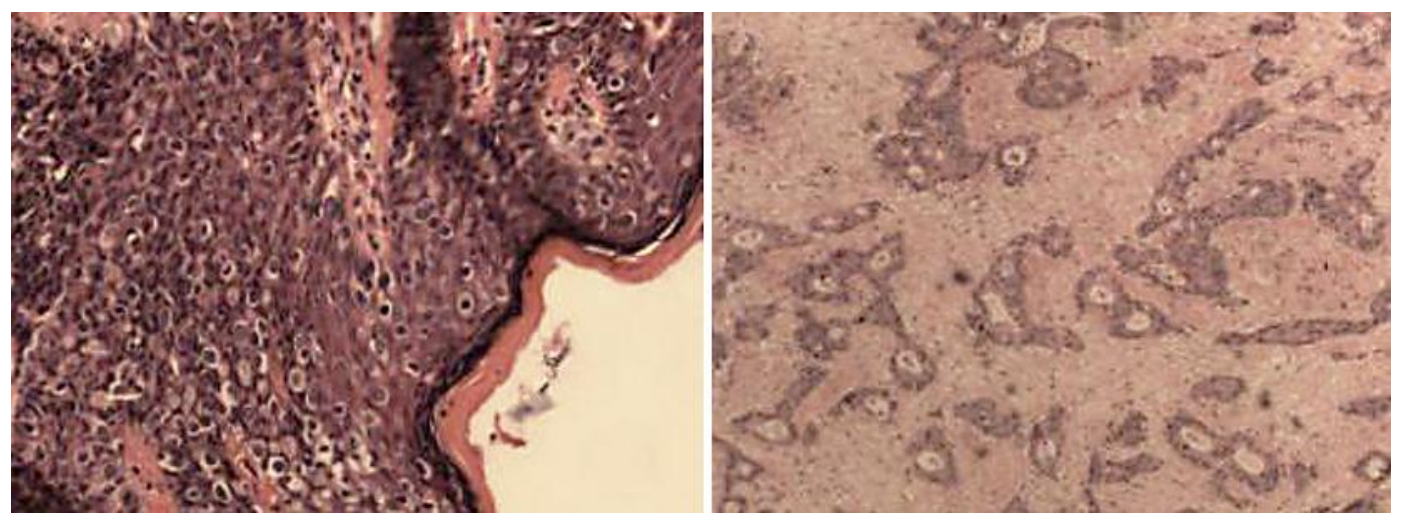

Fig. 1. Pathological images. Left: Histopathological image of PPD $(H \& E, \times 100)$. Right: Histopathological image of rectal adenocarcinoma $(\mathrm{H} \& \mathrm{E}, \times 100)$. 

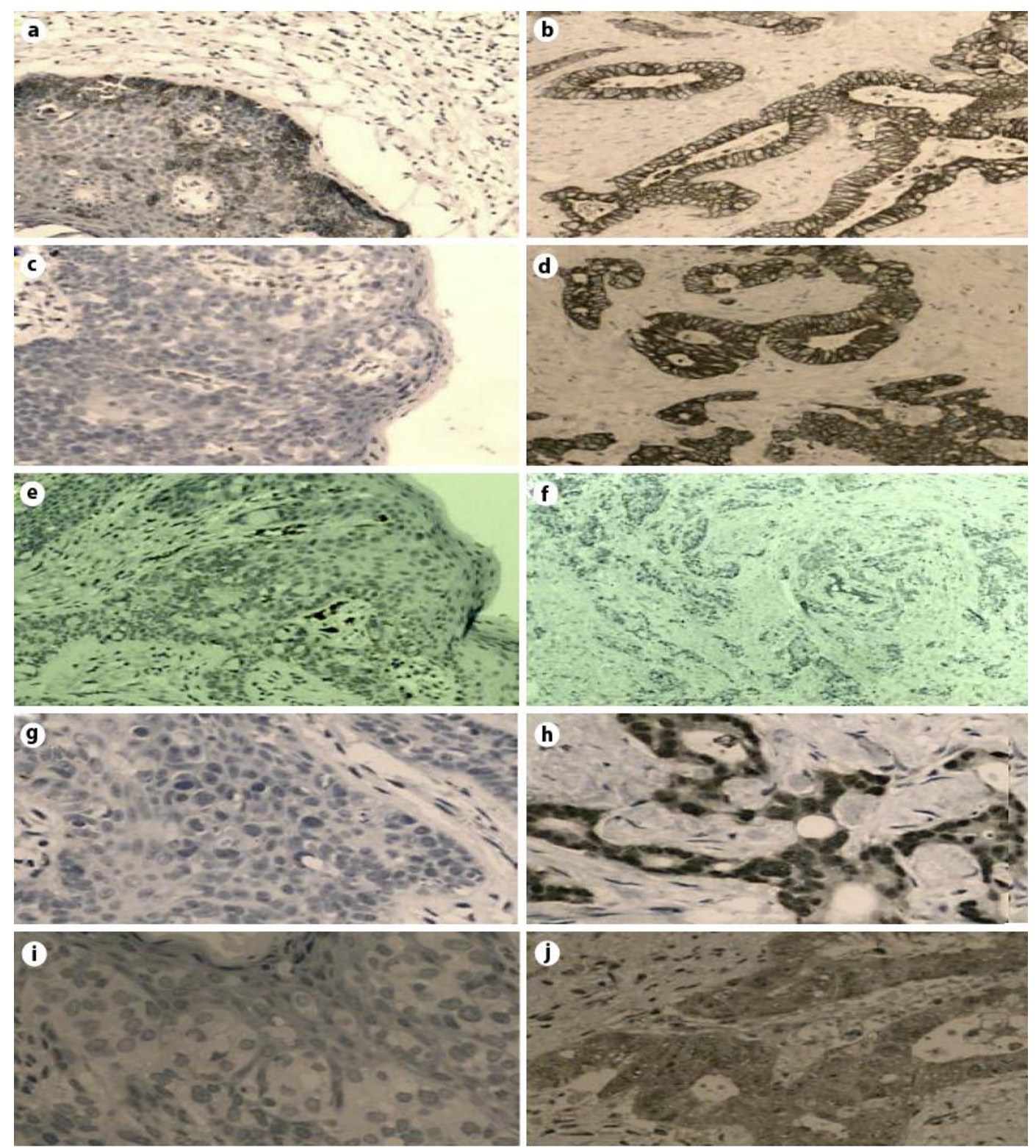

Fig. 2. Immunohistochemical analysis of the PPD and the associated anorectal adenocarcinoma. The intraepidermal Paget's cells are positive for CK7 (a) and negative for CK20 (c), GCDFP-15 (e), CDX2 (g) and MUC2 (i). On the other hand, the anorectal adenocarcinoma is positive for CK7 (b), CK20 (d), CDX2 (h) and MUC2 (j) and negative for GCDFP-15 (f). 